\title{
Les groupes sanguins des équidés du Tchad ${ }^{*}$
}

\author{
par $M^{\text {me }}$ L. PODLIACHOUK ef R. QUÉVAL
}

Les recherches sérologiques sur les groupes sanguins des animaux ont conduit à la détermination, dans diverses espèces, d'« antigènes érythrocytaires » dont la connaissance offre le plus grand intérêt en zootechnie.

Les groupes sanguins et leur transmission héréditaire peuvent servir à l'identification des animaux, à l'exclusion de paternité ou de maternité douteuse ou erronée, à la distinction des vrais jumeaux. En outre, ils peuvent être également utiles dans le choix des donneurs pour la transfusion sanguine, au choix d'animaux pour la production de sérums thérapeutiques et permettre la prophylaxie de la maladie hémolytique néo-natale des animaux. Ces applications sont d'un intérêt pratique immédiat.

L'étude approfondie de la mosaïque antigénique permettra d'étudier les linkages entre gènes érythrocytaires et gènes déterminant des caractères intéressant la production animale. Les applications futures des groupes sanguins porteront alors sur le contrôle du degré d'« in-breeding », l'étude systématique des races, la prévision de certaines qualités zootechniques liées à la présence de certains antigènes sanguins d'où une nouvelle orientation de la sélection ef du croisement, enfin, la réalisation de greffes de tissus ou d'organes.

Les applications présentes et futures des groupes sanguins des animaux portant sur la génétique, la physiologie, la zoologie et la pathologie suffisent à montrer l'importance de la question.

Lorsqu'en 1900 le Professeur Karl LANDSTEINER découvrit les groupes sanguins $A, B, O$ chez l'homme, nombreux furent les chercheurs qui comprirent l'intérêt que présenterait l'étude des groupes sanguins des animaux. Parmi les animaux domestiques le cheval retint l'attention de divers auteurs.

Reçu pour publication : décembre 1960.

Revue Elev. Méd. vét. Pays trop., 1961, 14. no 1.
L'un de nous avec EYQUEM (1) a étudié les réactions d'isoagglutination entre les sérums et les globules rouges de chevaux lesquels furent classés en 4 groupes $O, A, B, A B$ mais il fut constaté un grand nombre d'exceptions.

D'autres techniques furent utilisées ef nécessitèrent l'emploi de sérums de mulets, d'ânes et de chevaux absorbés à l'aide de globules rouges de chevaux.

Ces études ont permis d'invidualiser 11 antigènes érythrocytaires spécifiques des Equidés et qui ont été désignés par des lettres de l'alphabet dans l'ordre chronologique de leur découverte. Cette nomenclature n'a aucun rapport avec celle d'autres auteurs dans le même domaine ni avec celle des groupes sanguins de l'Homme (2),

Les antigènes $A, C, D, E, F, G, H, I$, J et $K$ sont spécifiques de l'espèce Cheval, tandis que $B$ est spécifique de l'Ane. On peut les retrouver tous chez le Mulet ou le Bardot.

L'étude de la répartition des antigènes érythrocytaires chez les chevaux suivant la race a été abordée antérieurement par plusieurs auteurs comme SCHERMER (1928), HOFE (1934) et LEHNERT (1939) (3). Comme chacun de ces auteurs utilisait une nomenclature qui lui était propre, la comparaison des résultats obtenus par chacun d'eux s'avère impossible.

La répartition des antigènes globulaires a été étudiée par l'un de nous (3) chez 712 chevaux de l'Annexe de l'Institut Pasteur de Garches (animaux appartenant à des races diverses), chez 47 chevaux de race ardennaise et chez 162 pur-sang.

La fréquence des divers antigènes érythrocytaires varie sensiblement avec la population étudiée. Ainsi la fréquence la plus élevée de l'antigène $D$ a été observée dans la race ardennaise $(0,51)$; chez les chevaux de Garches elle n'est que de 0,12 et 0 chez les pur-sang. La fréquence de l'antigène $E$ qui est de 0,31 chez les chevaux de Garches et de 0,20 chez les pur-sang, n'est que de 0,02 dans la race ardennaise.

* Cet article a paru, sous une forme un peu différente dans Ann. Inst. Pasteur, 1961, 100 (1) : 133-6. 
Depuis, à notre connaissance, seul SIRBU (8) en Roumanie a étudié la répartition des antigènes érythrocytaires équins dans 6 races différentes, désignéés dans son ouvrage sous les noms cidessous: Trotteur, Nonius, Arabe, Pur Sang galop, Ardennaise et Commune. II a effectué cette étude à l'aide de 6 agglutinines isolées par lui, déterminant les facteurs $A, B, C, D, E$ et $F$.

Pour pouvoir comparer les résultats des divers auteurs, il a fallu d'abord identifier les antigènes, la nomenclature de chaque auteur étant personnelle. Dans ce but l'un de nous et SIRBU ont procédé à un échange de sérums de référence, ce qui a permis l'identification de 5 antigènes de SIRBU avec ceux obtenus à I'Institut Pasteur (5).

Ainsi la comparaison de répartition des antigènes érythrocytaires équins en Roumanie et en France a permis de constater que la fréquence du facteur $D$ dans la race ardennaise et chez les pur-sang, est semblable à celle observée en France : elle est la plus forte chez les premiers et nulle chez les seconds. Les facteurs $A$ et $B$ de SIRBU, qui correspondent à nos $A$ et $C$, sont les plus fréquents dans toutes les populations étudiées par SIRBU ef l'un de nous.

L'étude des groupes sanguins des Equidés (chevaux et ânes), provenant de la République du Tchad (Afrique centrale), nous a donné les premiers résultats ci-dessous.

\section{CHEVAUX}

La presque totalité de la population chevaline de la République du Tchad dérive de deux grandes races originelles: aryenne ef mongolique avec des caractères ethniques assez purs chez certains sujets, mélangés en proportions variables chez d'autres ef chez la plupart avec des caractères de dégénérescence accusés dus au milieu et à l'Homme. Cependant on différencie 4 types: Arabe, Barbe, Dongola ef Poney Kirdi du Logone.

Ainsi le cheptel chevalin constitue une population assez peu homogène, même dans une seule région les divers types étant le plus souvent métissés entre eux. Cette hétérogénéité est la résultante de croisements en variation désordonnée, intensifs et inconsidérés $(6,7,9)$.

Notre première étude porte sur 59 de ces chevaux. La répartition des facteurs érythrocytaires est présentée au tableau $\mathrm{l}$.
TABLEAU 1


A $\ldots \ldots \ldots \ldots, 0,86$
0,75
C . . . . . .
0,86
D $\ldots \ldots \ldots \ldots, 0,02$
0,12
E $\ldots \ldots \ldots \ldots, 0,17$
0,31
F.......... 0,92
0,81
$H \ldots \ldots \ldots, 0,20$
0,16
J $\ldots \ldots \ldots \ldots, 0,27$
0,21

Ces 59 chevaux n'appartenant à aucune race définie, il nous semble que la population hétérogène des chevaux de Garches se prête le mieux à l'étude comparative de répartition des antigènes chez les chevaux du Tchad ef de la Métropole.

Le tableau I nous montre que la fréquence des divers facteurs globulaires chez les chevaux du Tchad ne diffère pas beaucoup de celle observée parmi les chevaux de Garches.

Les isoagglutinines sont rares dans le sérum des chevaux du Tchad examinés; fait déjà constaté lors de travaux antérieurs.

\section{ANES}

Les groupes sanguins des ânes ont été antérieurements éfudiés parl'un de nous avec EYQUEM(4). Au cours de cette étude qui a porté sur 135 mulets et 78 ânes, un seul antigène érythrocyłaire asin $B$ a pu être individualisé. La fréquence du facteur $B$ varie avec la race : elle est la plus élevée dans la race poitevine et très faible dans la race catalane. Chez les ânes de Paris, population hétérogène, la fréquence $d u$ facteur $B$ est de 0,62.

En Afrique équatoriale, la République du Tchad est pratiquement la seule à posséder des ânes. Ceux-ci constituent une population apparemment homogène. Ces animaux appartiennent tous au type unique si répandu en Afrique Noire: convexe, bréviligne, de petite taille $(0,90$ à $1,10 \mathrm{~m})$, à robe le plus souvent gris souris avec bande cruciale et raie de mulet. 
Notre étude a porté sur 31 ânes du Tchad. Le facteur érythrocytaire $B$ a été trouvé 18 fois : sa fréquence est donc de 0,58 .

La présence d'isoagglutinine anti-B dans le sérum de ces animaux est très fréquente. Elle a été décelée 7 fois dans le sérum de 13 ânes $B$ négatifs, tandis qu'au cours d'une étude précédente (1) sur les ânes de France et de Tunisie, elle n'a été décelée qu'une seule fois dans le sérum de 21 ânes $B$ négatifs.

En outre nous avons pu individualiser une nouvelle isoagglutinine naturelle spécifique de l'espèce asine. Nous l'avons dénommée anti- $M$; elle détermine le facteur $M$. Sur 25 ânes du Tchad examinés, 22 se sont révélés $M$ positifs et 3 seulement $M$ négatifs. Ainsi, la fréquence du facteur $M$ chez les ânes du Tchad est de 0,89. Chez 2 des 3 animaux $M$ négatifs, le sérum contient l'isoagglutinine anti- $M$.
En conclusion, cette étude préliminaire des groupes sanguins des Equidés du Tchad montre :

1) que la fréquence des facteurs érythrocytaires équins étudiés ne diffère pas beaucoup de celle observée dans la population hétérogène des chevaux de la Métropole.

2) laprésence d'isoagglutinines naturelles dans le sérum d'âne du Tchad est beaucoup plus fréquente que chez les ânes de la Métropole et de Tunisie, ce qui a rendu possible l'individualisation du second antigène érythrocytaire asin, nommé $M$.

Centre d'études des groupes sanguins des animaux, Loboratoire d'hématologie ef des groupes sanguins, Institut Pasteur (Paris) et

Laboratoire de recherches vétérinaires de Farcha Fort-Lamy (Tchad).

\section{RÉSUMÉ}

L'étude de 59 chevaux et de 31 ânes du Tchad a montré que la répartition des facteurs érythrocytaires chez les chevaux du Tchad ne diffère pas beaucoup de celle observée chez les chevaux de la Métropole. L'étude sérologique a permis d'individualiser un second antigène érythrocytaire asin, nommé $M$.

\section{SUMMARY}

\section{Blood groups in Horses of the Tchad Republic.}

In a study of 59 horses and 31 donkeys of Tchad it has been demonstrated that there is little variation in the disposition of the R. B. C. factors as between these animals and those of France. A serolofical study has revealed a second erythrocytic antigen in the donkey which has been designated $\ll M »$.

\section{RESUMEN}

Los grupos sanguíneos de los équidos del Tchad.

El estudio de 59 caballos y 31 asnos del Tchad ha mostrado que la relación de sus factores eritrocitarios no difiere gran cosa de la observada en los caballos de la metrópoli. El estudio serológico ha permitido individualizar un segundo antígeno eritrocitario en el asno, llamado $M$.

\section{BIBLIOGRAPHIE}

1. EYQUEM (A.) et PODLIACHOUK (L.), Groupes sanguins des équidés. IV. Nouvelles observations sérologiques. Ann. Inst. Pasteur, 1953, $85: 621-8$.
2. PODLIACHOUK (L.). - Les groupes sanguins des équidés (cheval, mulet, âne). Ann. Inst. Pasteur, 1958, $95:$ 7-22. 
3. PODLIACHOUK (L.). - Thèse de Doctoraf ès-Sciences, Paris, 1957.

4. PODLIACHOUK (L.) et EYQUEM (A.). Les groupes sanguins des équidés. III. Les groupes sanguins des ânes. Ann. Inst. Posteur, 1953, $84:$ 966-8.

5. PODLIACHOUK (L.), SIRBU (Z.), KOWNAC$K I(M$.$) et SZENIANSKA (D.). - Les groupes$ sanguins des chevaux. Etude comparative des sérums de référence. Ann. Inst. Pasteur, 1960. $98: 861$.
6. PECAUD (J.). - Monographie du Service de l'Elevage du Tchad (1926) (Non publiée).

7. RECEVEUR ( $\left.P_{1}\right)$. - Communication personnelle.

8. SIRBU (Z.). - Studiul serologic si genetic al grupelor sang-vine la cal in sase unitate de crestere. Inst. Path. Hyg. Anim. (Bucarest), 1959, 9 : 189-209.

9. THOMÉ (M.) - Communication personnelle. 